\title{
Coalescence and T-junction formation of carbon nanotubes: Action-derived molecular dynamics simulations
}

\author{
Sung Youb Kim, ${ }^{1}$ In-Ho Lee, ${ }^{2}$ Sukky Jun,${ }^{3}$ Youngmin Lee, ${ }^{1}$ and Seyoung $\operatorname{Im}{ }^{1}, *$ \\ ${ }^{1}$ Department of Mechanical Engineering, Korea Advanced Institute of Science and Technology, Daejeon 305-701, Korea \\ ${ }^{2}$ Korea Research Institute of Standards and Science, Daejeon 305-600, Korea \\ ${ }^{3}$ Department of Mechanical Engineering, University of Wyoming, Laramie, Wyoming 82071, USA
}

(Received 10 February 2006; revised manuscript received 20 September 2006; published 10 November 2006)

\begin{abstract}
The mechanisms of coalescence and T-junction formation of carbon nanotubes are analyzed using actionderived molecular dynamics. The control of kinetic energy in addition to the total energy leads to the determination of the minimum-energy atomistic pathway for each of these processes. Particularly, we find that the unit merging process of two carbon nanotubes consists of four sequential generalized Stone-Wales transformations occurring in four hexagon-heptagon pairs around the jointed part. In addition, we show that a single carbon atom may play the role of an autocatalyst, which significantly reduces the global activation energy barrier of the merging process. For $\mathrm{T}$ junction formation, two different models are chosen for simulation. One contains defects near the point of junction formation, while the other consists of two perfect nanotubes plus two additional carbon atoms. Our results indicate that the coalescence and junction formation of nanotubes may occur more easily than theoretically predicted in the presence of additional carbon atoms at moderate temperatures.
\end{abstract}

DOI: 10.1103/PhysRevB.74.195409

PACS number(s): 61.46.Fg, 81.07.De, 71.15.Pd

\section{INTRODUCTION}

Since their discovery, ${ }^{1,2}$ fullerenes and carbon nanotubes have been attracting growing interest as potential building blocks of nanoscale structures. The merging and junction formation mechanism of carbon nanostructures, which can broaden our understanding of the fabrication and manipulation of complex nanoscale devices and their components, have been widely investigated experimentally ${ }^{3-8}$ and theoretically. ${ }^{9-15}$ In experiments, the merging of fullerenes in a nanopeapod has been observed in electron beam irradiation $^{3}$ and studied under heat treatments. ${ }^{4}$ Two nanotubes can also form a larger one by electron beam irradiation ${ }^{5}$ or under thermal treatments without high energy particles, such as an electron beam. ${ }^{6,9}$ With the aid of computational resources, the process of fullerene fusion has been simulated by many researchers, including Zhao et al. ${ }^{10} \mathrm{Kim}$ et al., ${ }^{11}$ and Han et al. ${ }^{12}$ For the coalescence of two nanotubes, López et al. ${ }^{9}$ suggested a "patching and tearing" mechanism, wherein many initial defects like vacancies are presumed. Yoon et al. ${ }^{13}$ proposed a "zipping" mechanism, wherein two nanotubes can be merged only by successive generalized Stone-Wales (GSW) transformations of which the activation energy barriers are $5-6 \mathrm{eV}$.

However, as pointed out in early works, ${ }^{4,12,14}$ there is a wide gap between experimental observations and theoretical predictions in coalescence of fullerenes and nanotubes in terms of the energy barriers for these structural rearrangements. Under thermal treatment only, the fullerenes begin to merge with one another around $800{ }^{\circ} \mathrm{C}$ and complete coalescences below $1200{ }^{\circ} \mathrm{C} .{ }^{4}$ Nanotube fusion starts at $1800{ }^{\circ} \mathrm{C}$ and ends at $2200{ }^{\circ} \mathrm{C} .{ }^{6,9} \mathrm{In}$ these temperature conditions, the average thermal energies of carbon atoms are about $0.1 \mathrm{eV}$ for fullerenes and $0.2 \mathrm{eV}$ for nanotubes. The values of activation energy barriers obtained by theoretical approaches from first principles to empirical interatomic potential calcu- lations lie in the $5 \sim 7 \mathrm{eV}$ range. These theoretical predictions are not sufficient to explain why carbon nanostructures are merged so efficiently, even under relatively low temperatures. Though carbon atoms might, by chance, assume high energy with extremely small probability, it is hardly possible to overcome these high energy barriers, as indicated by Bandow et al. ${ }^{4}$

Autocatalysis studied in the Stone-Wales (SW) transformation of a fullerene, ${ }^{16}$ in dislocations in carbon structures, ${ }^{17}$ and in nanotube plasticity ${ }^{18}$ may provide us with a clue to bridge this gap. The presence of additional carbon atoms can be supported in a thermal decomposition environment ${ }^{7}$ and by the experimental deduction of migration of carbon atoms,${ }^{4}$ etc. Recently, Lee et al. ${ }^{14}$ reported that an additional carbon atom may play the role of a catalyst such that it may significantly reduce the energy barrier of fullerene fusion.

Since Zhou and Seraphin ${ }^{8}$ reported on junction structures of nanotubes, there have been experimental studies on these properties and on the methods of their mass production. ${ }^{19}$ Though there are many theoretical works ${ }^{15}$ on the electronic properties of the junctions with various shapes, the formation process has not been widely studied, other than the T-junction formation pathway that was proposed according to the topological changes possible with assumed initial defects on the models. ${ }^{20}$

In this work, we investigate the merging and T-junction formation procedures of nanotubes, including the effect of additional carbon atoms on these mechanisms using an action-derived molecular dynamics (ADMD) scheme. ${ }^{21,22}$ The ADMD formulation employed is introduced in Sec. II, and the results of the merging and junction formation mechanism are presented in Sec. III. The Conclusion follows in the final section.

\section{METHODOLOGY}

Regarding the simulation of rare event systems such as configurational changes of molecules, it is more favorable to 
optimize a specially designed object function connecting the given initial and final states than to execute ordinary molecular dynamics simulations until the system reaches the desired final state. ${ }^{23} \mathrm{ADMD}$, one of the methods of optimizing the object function based on a variational concept, proved effective for the calculations of complex and concerted structural transformations of carbon molecules. ${ }^{11,14,22}$ The object function of ADMD is constructed by discretized classical action and proper dynamic constraints connecting the given initial and final atomic configurations. ADMD does not yield real reaction pathways, but it is extremely useful in that it provides a minimum-energy pathway leading to the lowest energy barrier. ${ }^{21,22}$ With the aid of dynamic constraints incorporated with the action, we can find dynamic pathways close to the Verlet trajectories passing through the lowest saddle point located between the two potential-energy minima. Especially, the control of kinetic energy of the system gives us improved dynamic pathways and potential energy variation along the pathways, including those in a transition state during these microscopic structural changes. ${ }^{22}$

For our simulations, total energy conservation and kinetic energy control terms are added to the discretized action of the object function as below, ${ }^{22}$

$$
\begin{aligned}
\widetilde{\Theta}\left(\left\{\mathbf{r}_{j}\right\}, E ; T\right)= & S^{h}\left(\left\{\mathbf{r}_{j}\right\}\right)+\mu_{E} \sum_{j=0}^{p-1}\left(E_{j}-E\right)^{2} \\
& +\mu_{K} \sum_{I=1}^{N}\left(\left\langle K_{I}\right\rangle-\frac{3 k_{B} T}{2}\right)^{2} .
\end{aligned}
$$

where $S^{h}$ is the discretized action connecting the initial and final configurations, $E_{j}$ the total energy at the $j$ th step, $\left\langle K_{I}\right\rangle$ the time average of the Ith atom's kinetic energy, $E$ the target energy, $T$ the target temperature, $\mu_{E}$ and $\mu_{K}$ penalty parameters, and $k_{B}$ the Boltzmann constant.

Here the presence of the two penalty terms, multiplied by $\mu_{E}$ and $\mu_{K}$, changes the nature of the original action integral $S^{h}$ such that the solution of the variational problem $\delta \widetilde{\Theta}=0$ corresponds to minima, not to saddle points. The determination of proper values of the penalty constants $\mu_{E}$ and $\mu_{K}$ are problem-dependent and more or less empirical. In the present study, we thoroughly examine the sensitivity of the resulting numerical solutions to the variation of these constants to determine their proper values systematically. In addition, the target energy $E$ and the target temperature $T$ should be appropriately prescribed to obtain a meaningful solution. It requires some trial and error procedure to fix optimal values of the target energy and target temperature. In the next section, we demonstrate the procedure of determining all these constants for the case of coalescence of two nanotubes.

\section{NUMERICAL RESULTS AND DISCUSSION}

\section{A. Coalescence of two nanotubes}

First we examine the merging mechanism of nanotubes by conducting ADMD simulations for the so-called "zipper mechanism" studied by tight-binding molecular dynamics ${ }^{3}$ and by the string method. ${ }^{13}$ We employ the same Y-junction shapes as Yoon et al. ${ }^{13}$ for the given initial and final configurations; the upper part of this junction is a $(10,10)$ nanotube, and the lower part consists of two $(5,5)$ nanotubes. Note that the initial and the final configuration is determined in that the movement of the atoms participating in the reaction may be minimized. The jointed part of the Y junction, whose position at the final model is located one period lower than that at the initial one, contains one octagon at the center, and the eight sides of this octagon are shared by the neighboring four heptagons and four hexagons in an alternating manner. Structural change from the initial configuration to the final one comprises a unit-merging process of nanotube coalescence.

Before we present our final numerical results, it is important to be ensured that all the constants, including the penalty constants, the target energy, temperature, and time step size, are properly set so that reasonable invariance of the numerical solution with respect to moderate variation of these parameters is obtained. Therefore we go through a trial-anderror procedure to set the proper values of these parameters. For convenience of computation we demonstrate this procedure for the case of Tersoff potential. ${ }^{25,24}$

First, our test runs show that $5.0 \mathrm{fs}$ is an appropriate time step size. This value is slightly smaller than the step size employed for the simulation of the SW transformation by Lee et $a l .{ }^{22}$ With this time-step size, we conduct simulation over 100 time steps, so that the total duration of simulation is $0.5 \mathrm{ps}$ for this checking procedure.

Second, initial trial values of the target energy $E$ are set based on the potential energies of the initial and the final configuration, and the proper values should be greater than the peak value on the potential energy profile due to the presence of the kinetic energy term. Initially we begin with a large value of $E$, and then the profile of the target energy remains flat. We continue to decrease the value until we start to notice a deviation from a constant value of this target energy. With the choice of the target temperature $T=500 \mathrm{~K}$ and the penalty parameters $\mu_{E}=10^{8}$ and $\mu_{K}=10^{10}$, Fig. 1(a) shows the profiles of the target energy and the potential energy versus step index or time, depending on various values of the target energy. We notice that a small deviation from a uniform value of the target energy starts to occur at around $E=-2262.5 \mathrm{eV}$. As far as the target energy is slightly greater than the peak value of the potential energy, we notice the invariance of the potential energy profile.

Third, we examine the solution invariance with respect to the variation of the target temperature. Figure 1(b) shows the profiles of the potential energy depending on the target temperature when $E=-2262.5 \mathrm{eV}$ and $\mu_{E}=10^{8}$ and $\mu_{K}=10^{10}$. We see that the energy profile remains almost invariant for the three temperature values shown.

Next, we explore the sensitivity of the resulting profile of the potential energy to the change of the penalty parameters $\mu_{E}$ and $\mu_{K}$. For the fixed values of $E=-2262.5 \mathrm{eV}, T$ $=500 \mathrm{~K}$, and $\mu_{K}=10^{8}$, we plot the profile of the potential energy for three values of $\mu_{E}, 10^{6}, 10^{8}$, and $10^{10}$, in Fig. 1(c). This shows that the solution is nearly insensitive to $\mu_{E}$ in the range between $10^{6}$ and $10^{8}$. However, the energy profile abruptly deviates from the one obtained for the appropriate range of $\mu_{E}=10^{6} \sim 10^{8}$, if the penalty constant $\mu_{E}$ is greater than $\mu_{K}$, such as, $\mu_{E}=10^{10}$. On the other hand, too small $\mu_{E}$ 


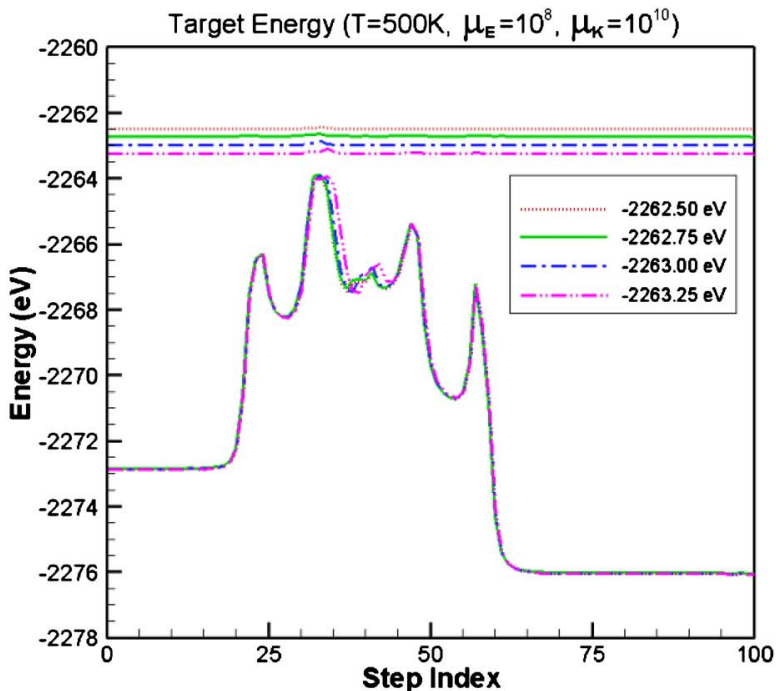

(a)

Penalty parameter $\mu_{E}\left(E=-2262.50 \mathrm{eV}, T=500 \mathrm{~K}, \mu_{\mathrm{K}}=10^{8}\right)$

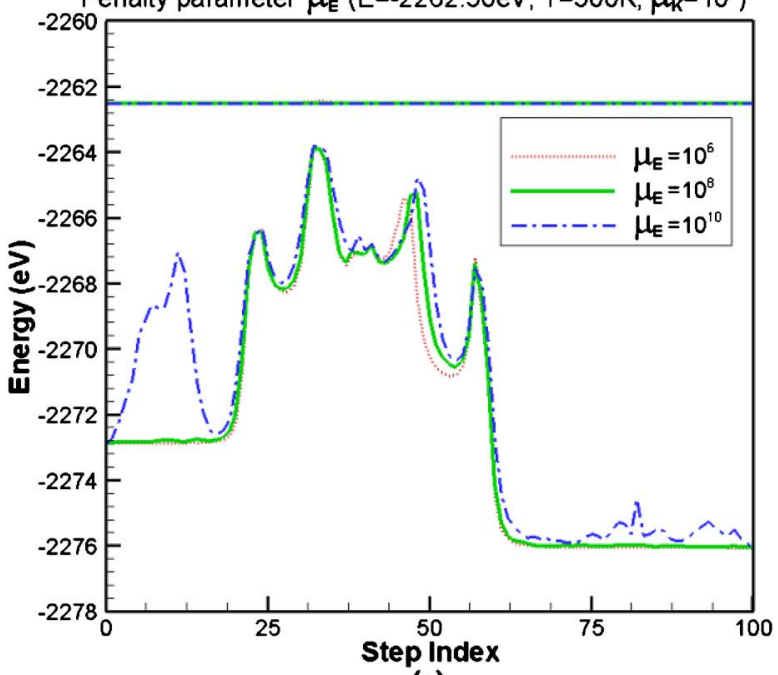

(c)

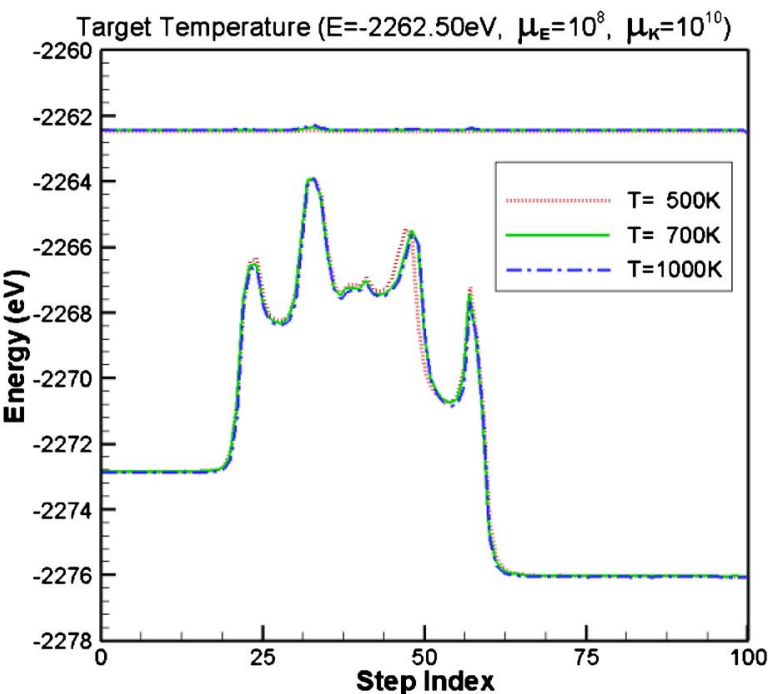

(b)

Penalty parameter $\mu_{\mathrm{K}}\left(E=-2262.50 \mathrm{eV}, T=500 \mathrm{~K}, \mu_{\mathrm{E}}=10^{8}\right)$

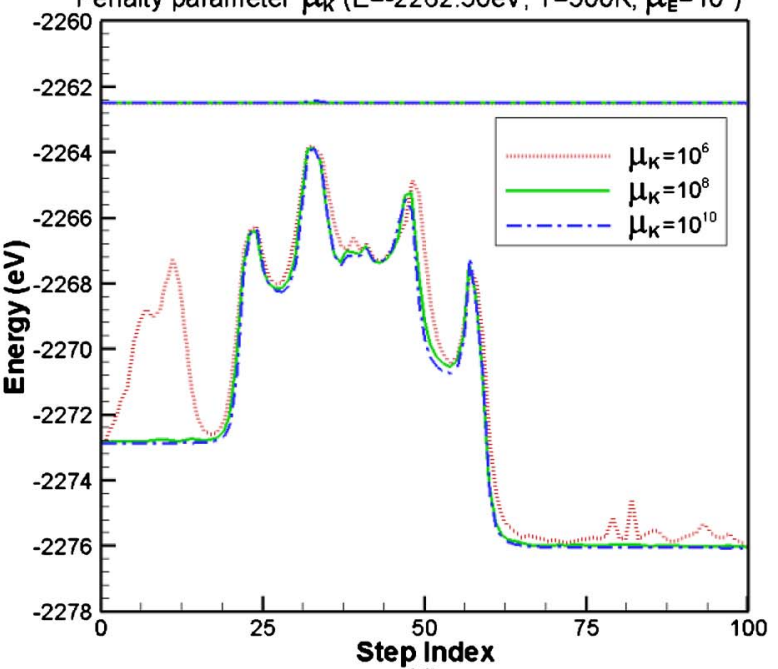

(d)

FIG. 1. (Color online) Profiles of the potential energy versus time (step index) for the coalescence of two carbon nanotubes (Tersoff potential). Depending on the target energy $E$ (a), the target temperature $T(\mathrm{~b})$, the penalty parameter $\mu_{E}(\mathrm{c})$, and the penalty parameter $\mu_{K}(\mathrm{~d})$.

compared with $\mu_{K}$, for example $\mu_{E} / \mu_{K}$ less than $10^{-3}$, fails to impose the energy constraint and so leads to an inappropriate numerical solution. In the same way, the solution sensitivity to the change of the penalty constant $\mu_{K}$ is checked. Figure 1(d) shows that the energy profile is almost invariant with respect to the change of $\mu_{K}$ except for too small a value of $\mu_{K}=10^{6}$. Taking all our numerical experiments together, we can say that the appropriate values of the penalty constants $\mu_{E}$ and $\mu_{K}$ are $10^{8} \sim 10^{10}$ for the present problem and that $\mu_{K}$ is not smaller than $\mu_{E}$.

Finally, we have checked the sensitivity of the energy profile for the changes of various combinations of the parameters other than those aforementioned, and the results show that the numerical solution remains almost invariant unless some extreme values of the constants are chosen. In all of our subsequent simulation, we go through such thorough numerical tests to confirm that our numerical solutions are not sensitive to the choice of the parameters within the appropriate range.
After confirming the invariance of the numerical solutions through the aforementioned procedure, we have conducted simulation on the coalescence of carbon nanotubes in real earnest. The number of atoms is 324 , and the step size of $5.0 \mathrm{fs}$ as determined above is employed. The total simulation time is now extended to $1.0 \mathrm{ps}$ and the corresponding number of intervals is 200. We set the target energy to be about $9 \mathrm{eV}$ higher than the potential energy of the initial model, and the target temperature to $500 \mathrm{~K}$. The penalty parameters employed are $10^{8}$ for $\mu_{E}$ and $10^{10}$ for $\mu_{K}$. These values have been successfully employed by Lee et al. for simulation of the SW transformation. ${ }^{22}$

The dynamic structural changes during a unit-merging process are given in Fig. 2(a) by nine snapshots of the front view, including the initial and final configurations. The number below each snapshot indicates the corresponding sequence or step index on the potential energy profile of the merging process in Fig. 2(b). Four single-bond rotations, all of which are GSW transformations taking place in the red 

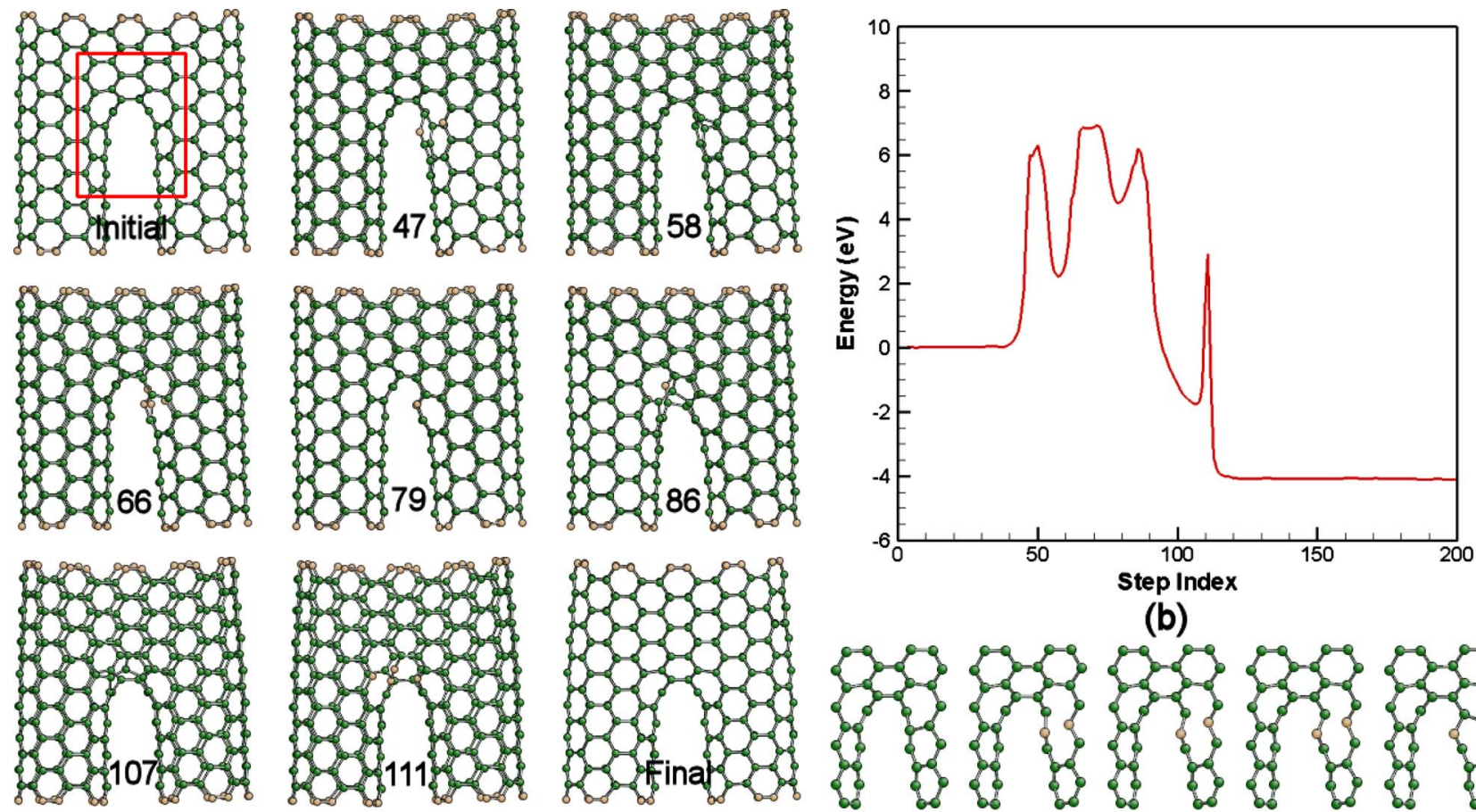

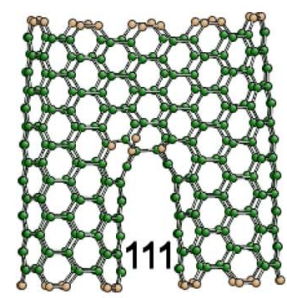

(a)
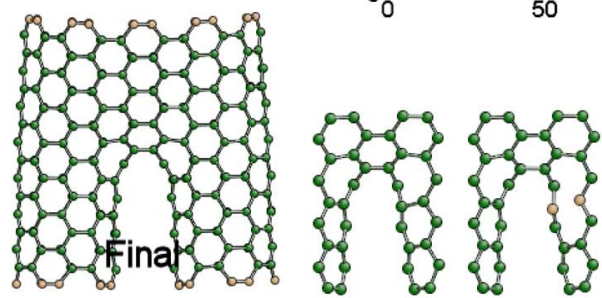

(b)

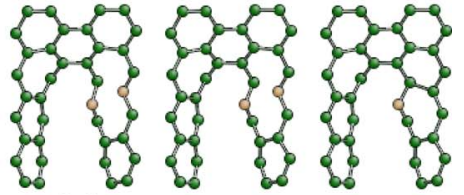

(c)

FIG. 2. (Color online) The result from the ADMD calculation for a unit merging process of nanotubes. Snapshots for structural changes with corresponding step index (a) and a potential energy profile of the obtained dynamic trajectories using the tight-binding potential (b). The four main peaks indicate the GSW transformations. Each GSW occurs in a heptagon-hexagon pair, and the first one is given by zoomed snapshots in (c).

rectangle in Fig. 2(a), are observed during the unit-merging step, and the potential energies of their transition states are indicated by the four main peaks in the potential energy curve. The activation energy barriers of each GSW transformation are 5.9, 4.5, 2.0, and $3.5 \mathrm{eV}$ for Tersoff potential and $6.3,4.7,1.7$, and $4.6 \mathrm{eV}$ for tight-binding potential ${ }^{25}$ in order of time sequence. The first two peaks in Fig. 2(b) show that GSW transformations are multistep processes, as reported earlier in the SW transformation. ${ }^{22,26}$ The global activation energy barrier, which is defined by the difference between the highest potential energy and the initial one, is $8.9 \mathrm{eV}$ for Tersoff, and $6.9 \mathrm{eV}$ for tight-binding potential. The first GSW transformation is depicted in Fig. 2(c), with five zoomed snapshots of which the step indices correspond from 45 to 49.

Each GSW transformation occurs between each of the four heptagons and its adjacent hexagon below in Fig. 2. The GSW transformations result in breakage of two bonds on the octagon at the center, and this gives rise to the formation of a new octagon, which completes one unit-merging step. To facilitate comprehension, we depict the details of the joining part in Fig. 3, wherein GSW transformations occur. We set four coordinate axes along the symmetric lines of the model, as described in Fig. 3(a) with Greek letters, and label some carbon atoms participating in GSW transformations with lower-case letters. We also number the polygons around the joining part in counterclockwise manner from the bottom view. Some of them are not shown in Figs. 3(b) and 3(c), because they are located in the back side of the model. Two schematic development figures around the joining part, be- fore and after GSW transformations, respectively, are drawn in Figs. 3(d) and 3(e) from the bottom view. The first bond rotation, occurring on the bond between atom $a$ and atom $b$ (sharing bond of heptagon 1 and hexagon 2), is apparently a GSW transformation. Bonds between atoms $a$ and $c$, and atoms $b$ and $h$ are broken due to the rotation of the bond $a-b$. Finally, atom $a$ rebonds with atom $e$, and atom $b$ with atom $c$. During this reaction, the system should overcome approximately $\sim 6 \mathrm{eV}$ of energy barrier. Other bond rotations take place in bonds $e-f, i-j$, and $m-n$ in order of time sequence, and finally a new octagon is formed at the center with atoms $a, e, h, l, i, m, p$, and $d$. We indicate the new bonds formed as a result of the GSW transformations with dotted lines in Fig. 3(e). It is noted that the process is completed only through the bond rotations of the four sharing bonds on the four heptagon-hexagon pairs. The visualization of the complete merging process is presented online (see Ref. 27).

\section{B. The effect of an additional carbon atom}

In order to investigate the effects of an adatom, we include a single carbon atom to the previous model and conduct six cases with different initial and final positions of the extra atom. The same conditions and parameters as the previous cases are used. One of the results is shown in Fig. 4. The dynamic structural changes are given in Fig. 4(a) by nine snapshots, including the initial and final configurations. An extra carbon atom is located at the front side of the system in the initial configuration, and it moves toward the back 


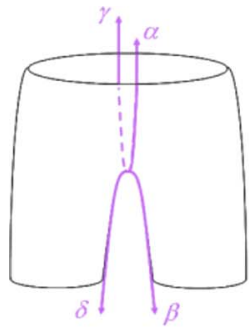

(a)
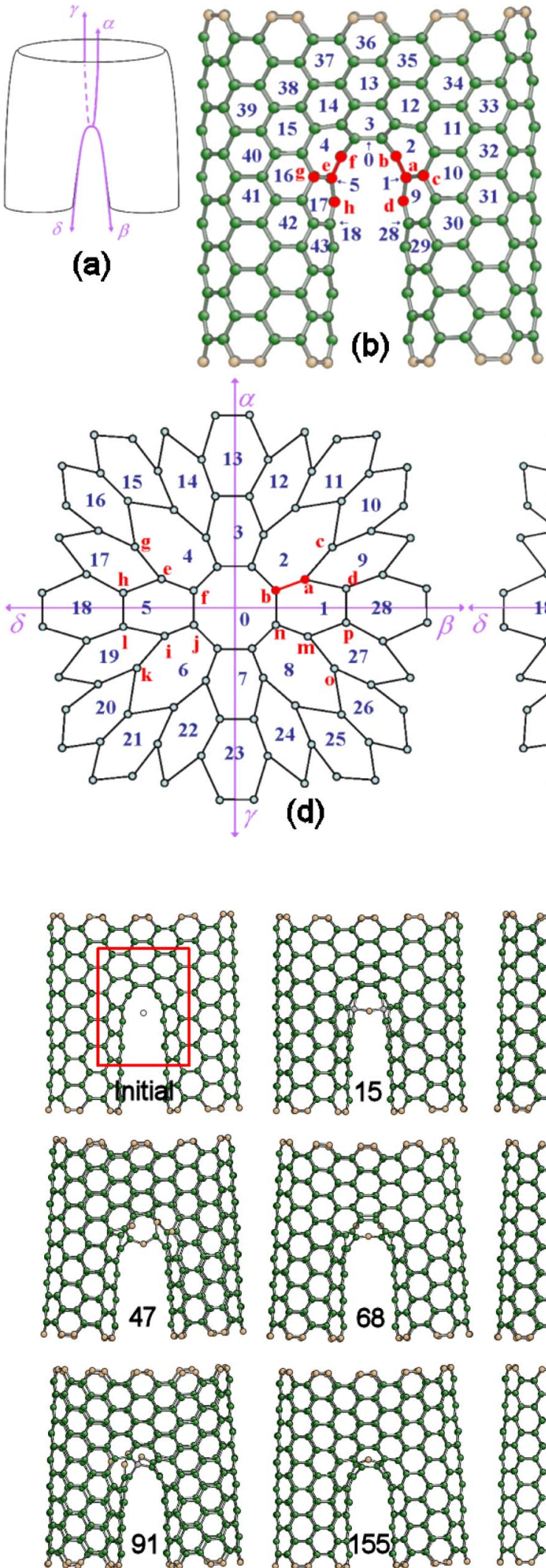

(d)
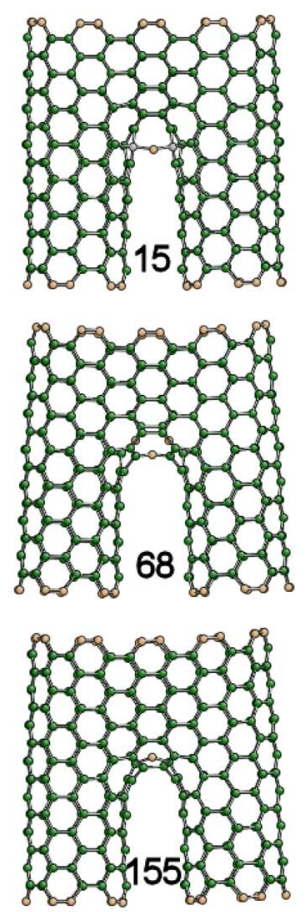

(a)

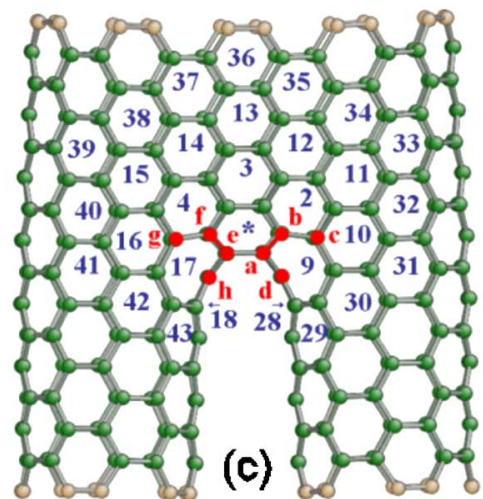

(c)

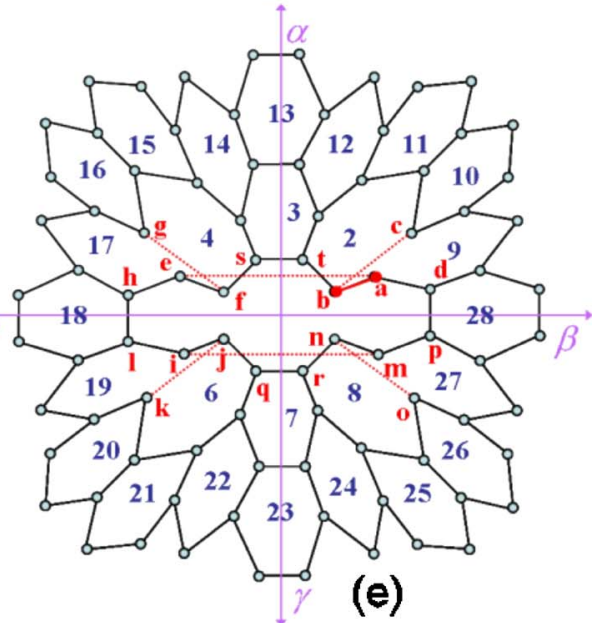

FIG. 3. (Color online) The occurrence positions of GSW transformations. Coordinate axes for development figures are set as in (a). Atomic configurations from front view are shown in (b) and (c). Numbers indicate polygon and small letters indicate with the GSW transformations. Development figures from bottom view before and after GSW transformation are in (d) and (e), respectively. Bond $a-b$ is rotating. New bond formed after GSW transformations are presented with dotted lines in (e). See text for details.
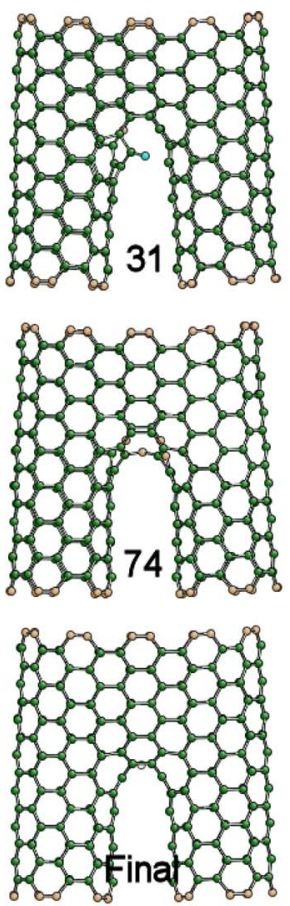

Finatson

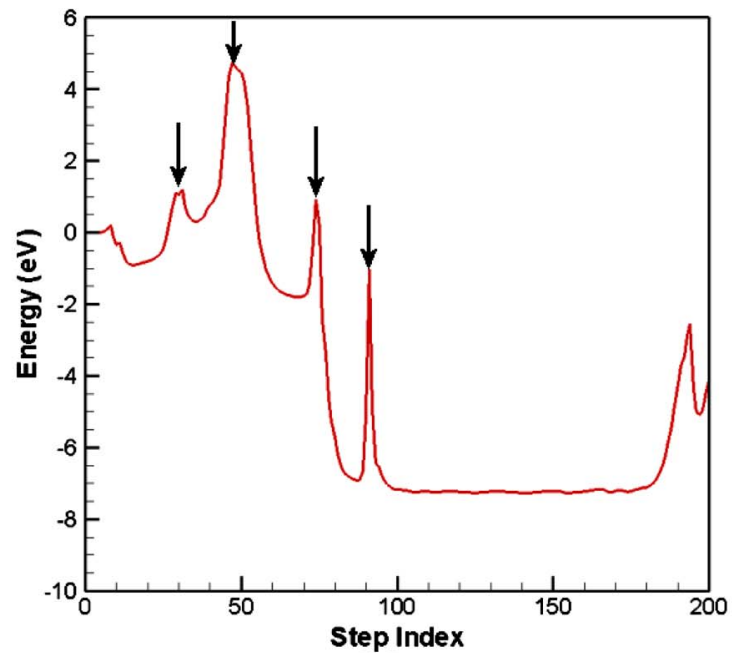

(b)

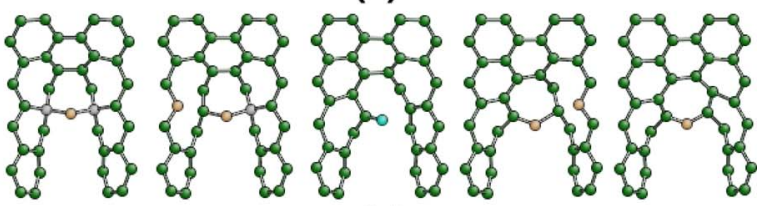

(c)

FIG. 4. (Color online) A single carbon atom assists the unit merging process. Snapshots for structural changes, including a carbon atom with corresponding step index (a) and a potential energy profile using the tight-binding potential (b). The four peaks indicated by arrows, corresponding to the four GSW transformations, are similar to the uncatalytic case. The first two GSW transformations, which occurred in the front part of the $\mathrm{Y}$ junction, are given by zoomed snapshots in (c). 


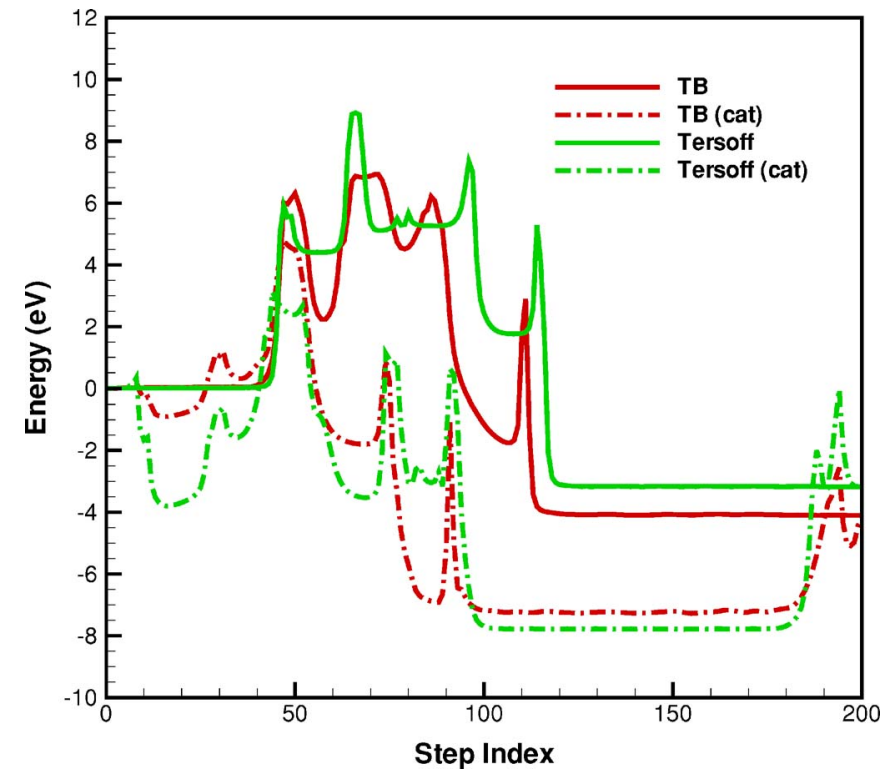

FIG. 5. (Color online) Potential curves during the unit merging process with and without an additional carbon atom.

side of the system, finally escaping from the $\mathrm{Y}$ junction. Because the $s p^{2}$ bond in a part of the system is changed to an $s p^{3}$ bond due to the extra atom, the procedure in this case differs from that in the previous one. Before step index 20, the additional carbon atom forms bonds with the two atoms on both sides of the $\mathrm{Y}$ junction, as shown in the second snapshot (step index 15) in Fig. 4(a). The atom making a bond with the adatom is located at the sharing bond, in which the GSW transformation occurs, of a heptagon and its neighboring hexagon below. After bonding, the additional carbon atom assists two GSW transformations that occur between two hexagon-heptagon pairs located at the front part of the Y junction (step indices from 20 to 69). This is followed by the other two GSW transformations taking place at the back part of the $\mathrm{Y}$ junction, which correspond to step indices from 70 to 101 . The last peak at around step index 194 on the potential curve indicates the energy barrier for the adatom to escape from the $\mathrm{Y}$ junction by bond-breaking. Two GSW transformations occurring at the front part of the system are shown in Fig. 4(c), with five zoomed snapshots of the part indicated by a rectangle on the initial configuration.

A unit-merging process of nanotubes in the presence of the adatom consists of four GSW transformations as well, and the positions in which the GSW transformations occur remain the same as the noncatalytic case. However, the additional carbon atom changes the sequential order of GSW transformations and reduces the global activation energy barrier from $8.9 \mathrm{eV}$ to $3.0 \mathrm{eV}$ for Tersoff potential and from $6.9 \mathrm{eV}$ to $4.8 \mathrm{eV}$ for tight-binding potential, as shown in Fig. 5. For both potentials the additional atom plays the role of an autocatalyst, reducing the global energy barrier. However, the effect is more apparently revealed in the case of Tersoff potential. The reduction of global activation energies due to an additional carbon atom during the merging process may explain its experimental observations under mild temperature conditions.

\section{T-junction formation pathways}

Next, we present the T-junction formation processes with two different initial configurations: one contains a defected nanotube and the other includes the model of the perfect one in addition to two carbon atoms. For a comparative study, we choose the same model that was used for the topological study of finding formation pathways ${ }^{20}$ for the first case. In this model, a $(5,5)$ nanotube containing initial defects is positioned at the upper part, and a $(10,0)$ nanotube capped by half of the fullerene is located at the lower part. The $(5,5)$ nanotube contains two more carbon atoms than the corresponding one free from defects, and $s p^{2}$ bonds are maintained throughout the process. In the latter model, a perfect $(5,5)$ nanotube is used instead of a defected one at the same position with two additional carbon atoms, as shown in the first snapshot of Fig. 6(b). These two atoms are needed to form the T-shaped junction of nanotubes. The potential profiles of both cases during T-junction formation are given in Fig. 6(a). Whereas the global activation barrier of the former case was about $3 \mathrm{eV}$ in the topological study, ${ }^{20}$ it is nearly zero along the dynamic pathways we have obtained. In the early steps, four atoms at the cap of the $(10,0)$ nanotube, after cap opening, make bonds with the atoms in the defected part of the $(5,5)$ nanotube. This process, whose step index ranges

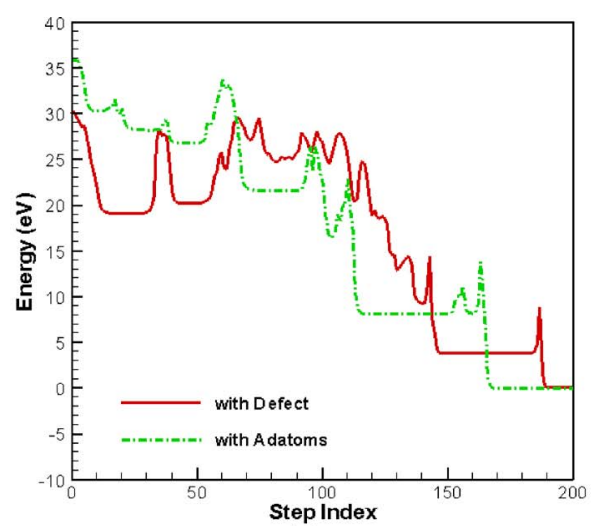

(a)

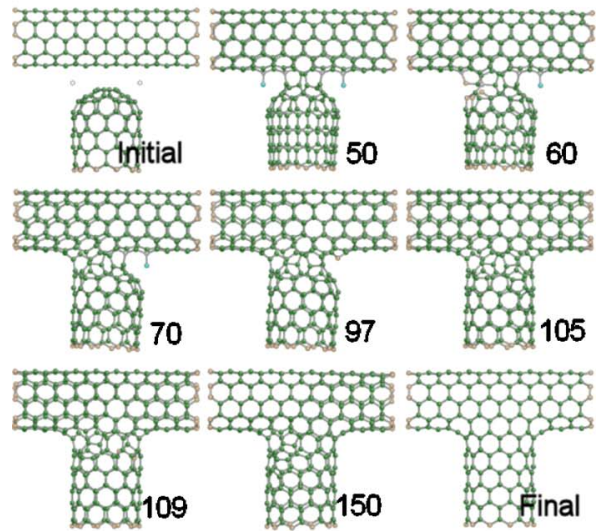

(b)

FIG. 6. (Color online) Formation processes for $\mathrm{T}$ junction of nanotubes. The potential energy profiles of two different initial configurations using Tersoff potential (a) and snapshots of structural changes during the junction formation from a $(5,5)$ and a capped $(10,0)$ nanotube, including two additional carbon atoms (b). 
from 0 to 20, lowers the potential energy of the system more than $10 \mathrm{eV}$. The complex bond-rotating, bond-breaking, and rebonding events between carbon atoms occur during step indices from 50 to 135 , and most of them are GSW transformations. In the latter model, the reaction process of T-junction formation is almost barrierless as well. The formation pathways are depicted in Fig. 6(b) by nine snapshots with the corresponding step indices, including the initial and final configurations. The process of cap opening and creating four bonds with a $(5,5)$ nanotube is similar to the former case during the early steps of junction formations. The reduction of the potential energy of the system is approximately $9 \mathrm{eV}$. After step index 52, the additional atoms assist structural changes which are represented by the four main peaks in the potential energy profiles in Fig. 6(a). The visualization of the complete formation processes are presented online (see Ref. 27).

Both processes of the T-junction formation are exothermic reactions with nearly negligible energy barriers, though they have different initial configurations. However, it should be noted that the possibilities of the events for realizing this process in experiment, among which are alignment of the end of a nanotube to the side of another one, the generation of the initial defects on the nanotube, and/or the existence of additional atoms at a proper distance, may be extremely low. Our results show that the reaction rate of junction formations depends more on these possibilities rather than on the activation energy barriers.

\section{CONCLUSIONS}

In conclusion, via action-derived molecular dynamics simulations we have shown that a unit-merging process of nanotubes consists of four GSW transformations which occur at the four hexagon-heptagon pairs on the Y-junction structures. Calculated activation energy barriers for GSW transformations are $6.3,4.7,1.7$, and $4.6 \mathrm{eV}$ for the tightbinding potential and 5.9, 4.5, 2.0, and $3.5 \mathrm{eV}$ for Tersoff potential in order of time sequence on time versus energy plots, and these values are comparable to SW-type transformations in other carbon nanostructures.

In the presence of an additional carbon atom, we observe that the global activation energy barrier of the merging step may decrease from $6.9 \mathrm{eV}$ to $4.8 \mathrm{eV}$ for the tight-binding potential, and from $8.9 \mathrm{eV}$ to $3.0 \mathrm{eV}$ for the Tersoff potential. This reduction of global energy barriers due to the autocatalytic role of the carbon adatom may be an explanation of the fast merging event of nanotubes at relatively low temperature in experiment.

We have presented the dynamic pathways of T-junction formation as well with two different initial configurations. In both cases, the activation energy barriers along the obtained dynamic pathways turn out to be negligible.

\section{ACKNOWLEDGMENTS}

This work was supported by a Korea Research Foundation Grant funded by the Korean Government (MOEHRD) (KRF-2005-041-D00029) for S.Y.K., Y.L. and S.I., and by the Ministry of Commerce, Industry, and Energy of Korea through the "R\&D Project for Key Technology" for I.H.L., and by "The 6th Strategic Supercomputing Applications Support Program" of the Korea Institute of Science and Technology Information.
*Author to whom correspondence should be addressed. Electronic address: sim@kaist.ac.kr

${ }^{1}$ H. W. Kroto, J. R. Heath, S. C. O'Brien, R. F. Curl, and R. E. Smalley, Nature (London) 318, 162 (1985).

${ }^{2}$ S. Iijima, Nature (London) 354, 56 (1991).

${ }^{3}$ B. W. Smith, M. Monthioux, and D. E. Luzzi, Nature (London) 396, 323 (1998); B. W. Smith, M. Monthioux, and D. E. Luzzi, Chem. Phys. Lett. 531, 31 (1999).

${ }^{4}$ S. Bandow, M. Takizawa, K. Hirahara, M. Yudasaka, and S. Iijima, Chem. Phys. Lett. 337, 48 (2001); S. Bandow, T. Hiraoka, T. Yumura, K. Hirahara, H. Shinohara, and S. Iijima, ibid. 384, 320 (2004).

${ }^{5}$ M. Terrones, H. Terrones, J.-C. Charlier, F. Banhart, and P. M. Ajayan, Science 288, 1226 (2000).

${ }^{6}$ K. Méténier, S. Bonnamy, F. Béguin, C. Journet, P. Bernier, M. Lamy de La Chapelle, O. Chauvet, and S. Lefrant, Carbon 40, 1765 (2002).

${ }^{7}$ E. A. Rohlfing, D. M. Cox, and A. Kaldor, J. Chem. Phys. 81, 3322 (1984).

${ }^{8}$ D. Zhou and S. Seraphin, Chem. Phys. Lett. 238, 286 (1995).

${ }^{9}$ M. J. López, A. Rubio, J. A. Alonso, S. Lefrant, K. Méténier, and S. Bonnamy, Phys. Rev. Lett. 89, 255501 (2002).

${ }^{10}$ Y. Zhao, B. I. Yakobson, and R. E. Smalley, Phys. Rev. Lett. 88,
185501 (2002); Y. Zhao, R. E. Smalley, and B. I. Yakobson, Phys. Rev. B 66, 195409 (2002).

${ }^{11}$ Y.-H. Kim, I.-H. Lee, K. J. Chang, and S. Lee, Phys. Rev. Lett. 90, 065501 (2003).

${ }^{12}$ S. Han, M. Yoon, S. Berber, N. Park, E. Osawa, J. Ihm, and D. Tománek, Phys. Rev. B 70, 113402 (2004).

${ }^{13}$ M. Yoon, S. Han, G. Kim, S. Lee, S. Berber, E. Osawa, J. Ihm, M. Terrones, F. Banhart, J.-C. Charlier, N. Grobert, H. Terrones, P. M. Ajayan, and D. Tománek, Phys. Rev. Lett. 92, 075504 (2004).

${ }^{14}$ I.-H. Lee, S. Jun, H. Kim, S. Y. Kim, and Y. Lee, Appl. Phys. Lett. 88, 011913 (2006).

${ }^{15}$ Z. Yao, H. W. Ch. Postma, L. Balents, and C. Dekker, Nature (London) 402, 273 (1999); A. Andriotis, M. Menon, D. Srivastava, and L. Chernozatonskii, Phys. Rev. Lett. 87, 066802 (2001); V. Meunier, M. B. Nardelli, J. Bernholc, T. Zacharia, and J.-C. Charlier, Appl. Phys. Lett. 81, 5234 (2002).

${ }^{16}$ B. R. Eggen, M. I. Heggie, G. Jungnickel, C. D. Latham, R. Jones, and P. R. Briddon, Science 272, 87 (1996).

${ }^{17}$ P. Jensen, J. Gale, and X. Blase, Phys. Rev. B 66, 193403(R) (2002).

${ }^{18}$ C. P. Ewels, M. I. Heggie, and P. R. Briddon, Chem. Phys. Lett. 351, 178 (2002). 
${ }^{19}$ J. Li, C. Papadopoulos, and J. Xu, Nature (London) 402, 253 (1999); B. C. Satishkumar, P. J. Thomas, A. Govindaraj, and C. N. R. Rao, Appl. Phys. Lett. 77, 2530 (2000).

${ }^{20}$ M. Menon, A. N. Andriotis, D. Srivastava, I. Ponomareva, and L. A. Chernozatonskii, Phys. Rev. Lett. 91, 145501 (2003).

${ }^{21}$ D. Passerone and M. Parrinello, Phys. Rev. Lett. 87, 108302 (2001).

${ }^{22}$ I.-H. Lee, J. Lee, and S. Lee, Phys. Rev. B 68, 064303 (2003); I.-H. Lee, S. Y. Kim, and S. Jun, Comput. Methods Appl. Mech. Eng. 193, 1633 (2004); I.-H. Lee, H. Kim, and J. Lee, J. Chem.
Phys. 120, 4672 (2004).

${ }^{23}$ R. E. Gillilan and K. R. Wilson, J. Chem. Phys. 97, 1757 (1992); R. Olender and R. Elber, ibid. 105, 9299 (1996).

${ }^{24}$ J. Tersoff, Phys. Rev. Lett. 61, 2879 (1988); 39, 5566 (1989).

${ }^{25}$ C. H. Xu, C. Z. Wang, C. T. Chan, and K. M. Ho, J. Phys. Condens. Matter 4, 6047 (1992).

${ }^{26}$ P. A. Marcos, M. J. Lopez, A. Rubio, and J. A. Alonso, Chem. Phys. Lett. 273, 367 (1997).

${ }^{27}$ http://me.kaist.ac.kr/ sim/eng/recent/cnt_zip.html 